\title{
Theoretical and experimental analysis of quantum path interferences in high-order harmonic generation
}

\author{
T. Auguste* and P. Salières \\ CEA-Saclay, IRAMIS, Service des Photons, Atomes et Molécules, F-91191 Gif-sur-Yvette, France
}

A. S. Wyatt, A. Monmayrant, ${ }^{\dagger}$ and I. A. Walmsley

Clarendon Laboratory, Parks Road, Oxford OX1 3PU, United Kingdom

E. Cormier

CELIA, CNRS-CEA-Université Bordeaux 1, 351 Cours de la Libération, 33405 Talence, France

\author{
A. Zaïr, ${ }^{\ddagger}$ M. Holler, A. Guandalini, F. Schapper, J. Biegert, ${ }^{\S}$ L. Gallmann, and U. Keller \\ Physics Department, ETH Zurich, CH-8093 Zurich, Switzerland
}

(Received 7 May 2009; published 10 September 2009)

\begin{abstract}
We present theoretical and experimental studies on quantum path interferences in high-order harmonic generation. Simulations of the single-atom response allow us to calculate the different quantum paths contributions; their relative phases and the resulting interferences can be finely controlled through the laser intensity that provides an efficient means for controlling the electron trajectories with an accuracy on the ten attoseconds time scale. Simulations of the macroscopic response demonstrate the need of spatial and spectral filtering of the harmonic beam in order to observe the interferences between the two shortest quantum paths. Our numerical results are in very good agreement with experimental data. These investigations represent a step toward the full characterization and control of the atomic harmonic dipole.
\end{abstract}

DOI: 10.1103/PhysRevA.80.033817

PACS number(s): 42.65.Ky, 32.80.Rm

\section{INTRODUCTION}

It is now well established that high-order harmonic generation (HHG) constitutes a very easy and robust method to produce attosecond extreme ultraviolet (xuv) emission by interaction of an intense laser field with an atomic or molecular target [1]. It is possible to generate trains of attosecond pulses [2-4] or isolated attosecond pulses [5,6]. These pulses are useful for the investigation of electron wavepacket dynamics with attosecond temporal resolution in atoms $[7,8]$ and in molecules [9]. The theoretical description of the HHG process has been first performed through the semiclassical three-step model $[10,11]$ : an electron initially in the ground state is freed into the continuum by tunnel ionization, accelerated, and driven back to the parent ion where it may recombine leading to the emission of an xuv photon. In order to describe the process more precisely, a quantum-mechanical theory has been developed within the strong-field approximation (SFA) $[12,13]$. In this model, the harmonic dipole moment is expressed as the coherent sum over all the different quantum paths contributing to the xuv emission. These quantum paths are a generalization of the classical electron trajectories described by the semiclassical model and account for quantum effects such as tunneling

\footnotetext{
*Corresponding author; thierry.auguste@cea.fr

†Present address: LAAS-CNRS, 31077 Toulouse, France.

Present address: Department of Physics, Blackett Laboratory, Imperial College London, South Kensington Campus, London SW7 2BW, UK.

${ }^{\S}$ Present address: ICFO, 08860 Castelldefels (Barcelona), Spain.
}

ionization and quantum diffusion. The dipole $d_{q}$-for the $q$ th harmonic order-can be written as

$$
d_{q}=\sum_{j} A_{q}^{(j)} e^{i S_{q}^{(j)}},
$$

where the summation is performed over the contributions from all possible quantum paths $j$, with weight $A_{q}^{(j)}$ and phase $S_{q}^{(j)}$. This phase is given by the quasiclassical action accumulated by the electron along the corresponding trajectory. It can be approximated by the product of the electron mean kinetic energy (the ponderomotive energy) times the excursion time, $S_{q}^{(j)} \approx-U_{p} \tau_{q}^{(j)} \approx-\alpha_{q}^{(j)} I$, where $I$ is the laser intensity. The longer the trajectory, the faster the intensity variation of the phase. The main contributing quantum paths correspond to a stationary action and are calculated by solving the saddle-point equations. They can be easily visualized through a Gabor-type analysis of the dipole where $\alpha_{q}^{(j)}$ is the reciprocal coefficient of the intensity [14]. This is illustrated in Fig. 1 where the $\alpha(I)$ distribution is plotted as a function of the laser intensity for the 21st harmonic calculated for argon within the SFA approach. At low intensity $I$ $<0.9 \times 10^{14} \mathrm{~W} / \mathrm{cm}^{2}$ (intensity corresponding to $I_{p}+3.17 U_{p}$ $=21 \omega$ ), there is one main contributing path corresponding to the cutoff trajectory $\tau_{q}^{\text {(cutoff) }} \approx 0.7 T$, with $T$ laser period, $\alpha_{q}^{(\text {cutoff })} \approx 13 \times 10^{-14} \mathrm{rad} \mathrm{cm}^{2} / \mathrm{W}$. For harmonics in the plateau region, the cutoff path bifurcates into two classes of trajectories, referred to as the short path, converging at high intensity to $\tau_{q}^{(1)} \approx T / 2, \alpha_{q}^{(1)} \approx 1-5 \times 10^{-14} \mathrm{rad} \mathrm{cm}^{2} / \mathrm{W}$, and the long path, converging to $\tau_{q}^{(2)} \approx T, \quad \alpha_{q}^{(2)} \approx 20-25$ $\times 10^{-14} \mathrm{rad} \mathrm{cm} / \mathrm{W}$. At $I>1.2 \times 10^{14} \mathrm{~W} / \mathrm{cm}^{2}$ (intensity corresponding to $\left.I_{p}+2.4 U_{p}=21 \omega\right)$, other branches appear at 


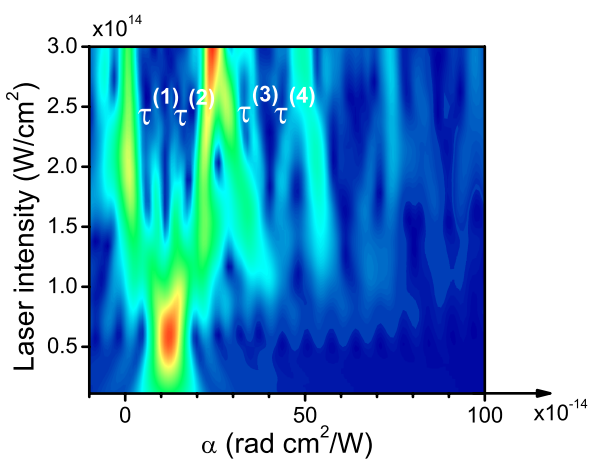

FIG. 1. (Color online) Calculated dependence of $\alpha$ with laser intensity resulting from a Gabor-type analysis of the dipole phase of harmonic 21 generated in argon.

large $\alpha$, corresponding to trajectories longer than 1 cycle that pass the nucleus more than once. Like the short and the long paths, they form "tuning forks" whose branches converge on either side to the branches of the neighboring tuning forks with increasing intensity. The strongest of these additional branches is the $\tau_{q}^{(3)}$ trajectory that converges to the $\tau_{q}^{(2)}$ path at high intensity. These longer trajectories have never been observed in high-harmonic generation up to now, presumably because their strongly intensity-dependent phase makes them very difficult to phase match. Note finally that while SFA accurately predicts the values of the corresponding $\alpha$, the weight of the different contributions may be quite different from the numerical solution of the time-dependent Schrödinger equation [15].

Due to their different phases, the contributions of the various quantum paths interfere and lead to a perturbed harmonic dipole. For instance, the relative phase of the contributions associated with two neighboring quantum paths $j$ and $j+1$ writes $\Delta \phi_{q}^{(j, j+1)} \approx-U_{p} \Delta \tau_{q}^{(j, j+1)} \approx-\Delta \alpha_{q}^{(j, j+1)} I$. This intensity dependence of the relative phase leads to modulations of the dipole moment with a period of $\delta I \approx 2 \pi / \Delta \alpha_{q}^{(j, j+1)}$. When the first pair of trajectories $(j=1,2)$ is involved in the process, the yield of the plateau harmonics exhibits fast oscillations with an average period of $0.3 \times 10^{14} \mathrm{~W} / \mathrm{cm}^{2}$, which is close to the expected $2 \pi / \Delta \alpha$ period of the quantum path interference (QPI) between the two first orbits (first-order QPI). In the case of $j=2,3, \Delta \alpha \approx 10 \times 10^{-14} \mathrm{rad} \mathrm{cm} / \mathrm{W}$ at $I=1.7$ $\times 10^{14} \mathrm{~W} / \mathrm{cm}^{2}$ and then it decreases with intensity. One thus expects that the interference period will increase with increasing intensity. Therefore, the interferences associated with each pair of trajectories have a specific behavior that allows their identification. Measuring all these quantum path interferences would permit one to fully characterize the atomic dipole. However, in the experiments, one gets access to the macroscopic harmonic field generated by a macroscopic ensemble of particles. The experimental conditions result in general in a spatial and temporal averaging over an intensity range much larger than $2 \pi / \Delta \alpha$ that smooths out the fast intensity modulations present in the single-atom response. This is the reason why even when contributions from the two main quantum paths have been independently characterized experimentally [16-20], only unmodulated yield distributions have been measured in the presence of multiple trajectories [21,22]. We recently obtained evidence of QPI by properly performing spectral and spatial filtering of the macroscopic signal in order to avoid temporal and spatial averaging [23].

In this paper, we study theoretically and experimentally the conditions needed for the observation of the QPI. We report a detailed numerical study as well as experimental measurements where the contrast is high enough to resolve the QPI over all the generated plateau harmonics in argon. This represents a step toward the direct experimental measurement and characterization of the full atomic dipole moment and the identification of the trajectories involved in the process. Moreover, due to the laser intensity control and the high stability of the laser system, we show that only tiny changes (of a few 10 as) of the electron excursion time are enough to shift the dipole relative phase by $\pi$, which corresponds to shifting from constructive to destructive QPI.

The paper is organized as follows. Section II is devoted to the simulation of the QPI in the single-atom and the macroscopic responses and to the presentation and discussion of the numerical results. In Sec. III, we describe the experimental setup and present the data obtained for different conditions of laser focusing and argon gas pressure. Finally, conclusions are given in Sec. IV.

\section{SIMULATIONS}

To get deeper insight into QPI, we carried out extensive numerical simulations. We first calculated the response of a single atom interacting with a strong laser field by solving the Schrödinger equation within the SFA [12]. We studied in particular the contribution of the different quantum paths to the interferences. In a second step, we simulated the macroscopic response of the generating medium in the different filtering conditions and studied the influence of the laser focus position and gas pressure. In this context, we also quantified the contribution of the quantum paths to the interferences.

\section{A. Single-atom response}

In order to analyze the QPI, we first calculated the dipole using the saddle-point method. In this case, the harmonic dipole is written as the coherent superposition of the contributions from the electron quantum paths evaluated at the (complex) saddle-point solutions [12,24]. The excursion times, entering the dipole phase defined in Sec. I and given by the solutions of the saddle-point equations, are plotted in Fig. 2 as a function of the laser peak intensity, for the 15 th harmonic and the two shortest trajectories (referred to as "short" and "long" in the figure). The excursion times vary quickly at low intensity, when the harmonic enters the plateau region and then much slowly.

Using the saddle-point method allows us to isolate the contribution of each trajectory to the dipole moment. The dashed and the dotted lines in Fig. 3(a) depict the dipole strength of the 21 st harmonic, calculated for argon, as a function of the laser intensity for the short and the long trajectories, respectively. Calculations are performed for an electric 


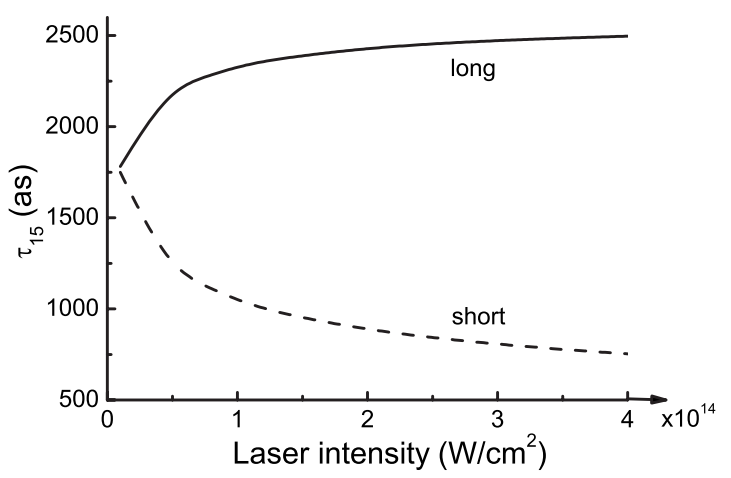

FIG. 2. Excursion time $\tau$ as a function of laser intensity calculated for the 15th harmonic and the two shortest trajectories.

field of the form $E(t)=E_{0} \cos (\omega t+\phi)$ [all along the paper, we suppose a carrier-envelope phase (CEP) equals to zero $(\phi$ $=0)$ ]. In both cases, the curve exhibits two regions with different slopes, corresponding to the cutoff and the plateau regions but there is no modulation. In contrast, when both trajectories are taken into account in the dipole calculation, interferences clearly appear. The harmonic dipole then exhibits fast oscillations with an average period of 0.3 $\times 10^{14} \mathrm{~W} / \mathrm{cm}^{2}$, which is close to the expected period of the quantum path interference between the two first orbits (firstorder QPI), as described in Sec. I.

A more accurate way to calculate the harmonic dipole is to perform the saddle-point integration only on the electron canonical momentum and to keep the full integration over

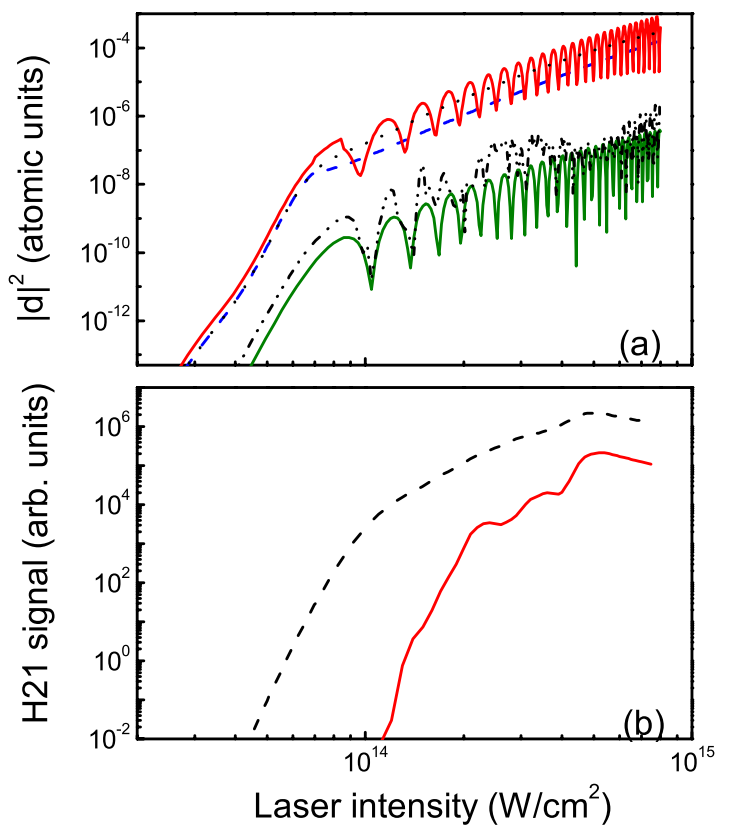

FIG. 3. (Color online) (a) H21 dipole strength versus laser intensity calculated for argon: using the saddle-point method (upper curves) for the short (dashed line), the long (dotted line), and the short+long (solid line) trajectories; using the SFA formula (lower curves) for the short+long trajectories (solid line) and all possible paths (dashed-dotted line) (the two latter curves are shifted downward for visibility). (b) Macroscopic $\mathrm{H} 21$ yield with (solid line) and without (dashed line) spatial filtering (see text). the two other variables: the excursion time $\tau$ and the recombination time $t$; this is the usual form of the (adiabatic) SFA [25]. When restricting the integration over $\tau$ to a single laser period [solid line (lower curves) in Fig. 3(a)], one basically selects the contributions of the two shortest paths and one recovers a dipole with similar QPI as observed on the solid curve (upper curves) of Fig. 3(a). When the $\tau$ integration is now performed over six periods, we include the contributions of higher-order trajectories, with return times in excess of one optical cycle (note that quantum diffusion effectively attenuates longer trajectories contributions). The dasheddotted line in Fig. 3(a) shows a very modulated behavior as soon as these longer trajectories come into play, i.e., above $1.2 \times 10^{14} \mathrm{~W} / \mathrm{cm}^{2}$ (corresponding to $I_{p}+2.4 U_{p}=21 \omega$ ) [25]. Many interference patterns with different periodicities are now superimposed corresponding to first-order and higherorder QPIs. Because of such fast oscillations, it is clear that the conditions to observe QPI will be difficult to find.

In Fig. 3, the dipole was calculated for fixed laser intensities. But in the experimental conditions, one can expect that the laser pulse temporal envelope will lead to temporal averaging and to reduced QPI contrast. In [23], we proposed to circumvent this problem by performing a spectral analysis of the harmonic dipole. Indeed, the temporal variation of the dipole phase induces a chirp of the harmonic emission through a phase modulation process $[15,26-30]$

$$
\Delta \omega(t)=-\frac{\partial \phi_{q}^{(j)}(t)}{\partial t} \approx \alpha_{q}^{(j)} \frac{\partial I}{\partial t} .
$$

This chirp, and thus the harmonic spectral bandwidth, increases with laser intensity. If one considers the signal precisely at the harmonic central frequency, it always corresponds in time to the maximum of the laser temporal envelope, while the emission from the envelope edges is shifted to different side frequencies: the harmonic chirp effectively maps time to frequency $\Delta \omega=\omega-\omega_{q}$. By spectrally resolving the harmonic, we should thus get rid of the temporal averaging.

We simulated the interaction of a $\sin ^{2}$ laser pulse of $30 \mathrm{fs}$ full width at half maximum (FWHM) with an argon atom. The spectra were calculated using the nonadiabatic SFA [31] in order to get the full harmonic spectrum. Note that we are not interested here in studying nonadiabatic effects since, in our experiments, the laser carrier-envelope offset phase (CEP) was not stabilized, as discussed in the experimental section below, so all the macroscopic simulations performed in the following will be adiabatic. In Fig. 4, we plot the spectra obtained when taking into account (a) the shortest trajectory alone and (b) the two first trajectories. This selection of trajectories was obtained by varying the time integration over $\tau$. As expected, when the two shortest trajectories are taken into account [Fig. 4(b)], the yield at each harmonic central frequency is modulated with a period consistent with QPI, and the harmonic spectral width increases. On either side of the central harmonic frequency, these interferences exhibit a parabolic shape with increasing intensity. The fringes are shifted to higher peak laser intensity when $\Delta \omega$ increases because these frequencies are generated on the 

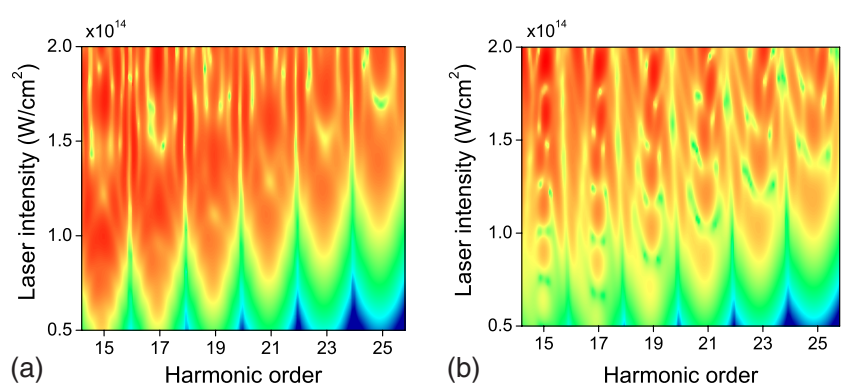

FIG. 4. (Color online) Single-atom spectra calculated within the nonadiabatic SFA. (a) Only the short trajectory is selected, i.e., time integration is performed over 0.65 optical cycle. (b) Integration time corresponds to the two first quantum paths.

edges of the laser temporal envelope and thus correspond to a smaller effective intensity. Even more complicated patterns are observed when higher-order trajectories (with return time in excess of half a cycle) are taken into account in the dipole calculation [23], due to higher-order QPI.

However, in the spectra calculated with only the shortest trajectory [Fig. 4(a)], slow spectral modulations already appear that cannot be attributed to QPI. Instead, these modulations are a direct consequence of the phase modulation that produces a different sort of interference. Indeed, similarly to self-phase modulation of an intense laser pulse propagating in a nonlinear medium, the corresponding spectrum may exhibit oscillations: each frequency may be generated at two different times in the temporal envelope on either side of the inflexion point, leading to interferences. Phase modulation and QPI are thus two independent sources of interferences that need further investigations in order to identify the actual origin of the modulations observed in the experimental data. Therefore, using a model dipole, we investigate the combined effects of temporal phase modulation and QPI. Assuming the first pair of quantum paths, the spectrum is given by

$$
S_{q}(\omega)=\left|F\left\{A_{q}^{(1)}(t) \exp \left[-i \alpha_{q}^{(1)} I(t)\right]+A_{q}^{(2)}(t) \exp \left[-i \alpha_{q}^{(2)} I(t)\right]\right\}\right|^{2},
$$

where $I(t)$ is modeled by a Gaussian function with $30 \mathrm{fs}$ FWHM, $\left|A_{q}^{(j)}(t)\right|^{2}=I(t), \alpha_{q}^{(1)}=3 \times 10^{-14} \mathrm{rad} \mathrm{cm}^{2} / \mathrm{W}, \alpha_{q}^{(2)}=25$ $\times 10^{-14} \mathrm{rad} \mathrm{cm}^{2} / \mathrm{W}$, and $F$ denotes the Fourier transform. In Fig. 5, we plot the spectral evolution with increasing peak intensity for quantum path (1) alone (top), quantum path (2) alone (center), and both paths (bottom). For each single path, the modulations are only due to phase modulations. A plot of the intensity dependence at the central frequency (right panel lower curves) clearly shows modulations with a periodicity of $2 \pi / \alpha$. For the long trajectory, this is close to the $2 \pi / \Delta \alpha$ periodicity of QPI, due to the small value of $\alpha_{q}^{(1)}$. However, these yield modulations disappear when the harmonic signal is spectrally integrated (upper curves on the right panel), in contrast to the QPI modulations that lose contrast but persist when integrated over frequency (bottom right panel). Note that the periodicity is exactly the same for the two curves in the latter plot (QPI) and differs slightly from that of the long path phase modulation (middle panel). This simplified model shows that spectral modulations are indeed due to both the

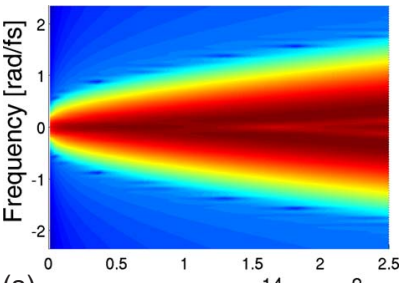

(a) Laser Intensity $\left[10^{14} \mathrm{~W} / \mathrm{cm}^{2}\right]$

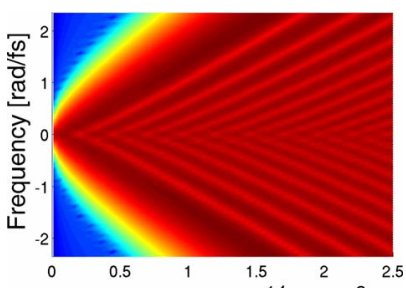

(c) Laser Intensity $\left[10^{14} \mathrm{~W} / \mathrm{cm}^{2}\right]$

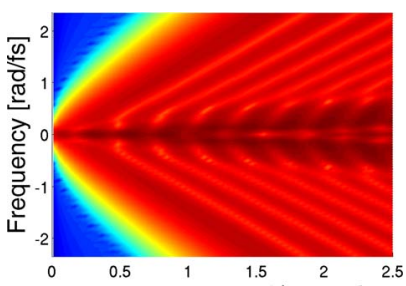

(e) Laser Intensity $\left[10^{14} \mathrm{~W} / \mathrm{cm}^{2}\right]$

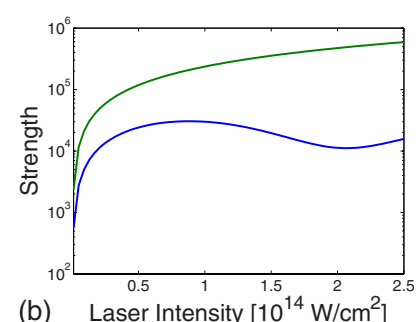

(b) Laser Intensity $\left[10^{14} \mathrm{~W} / \mathrm{cm}^{2}\right]$

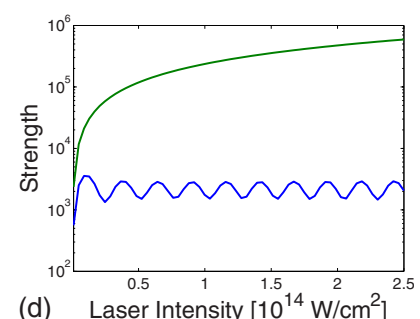

(d) Laser Intensity $\left[10^{14} \mathrm{~W} / \mathrm{cm}^{2}\right]$

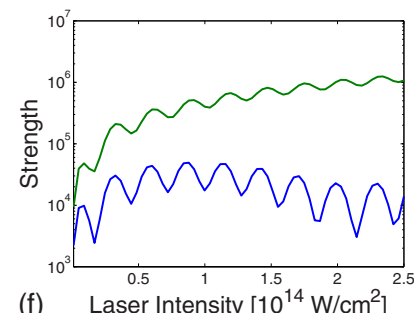

FIG. 5. (Color online) Harmonic spectrum evolution with laser intensity for a model dipole (left panels). Corresponding amplitude modulation at the central frequency (right panel lower curves) and integrated over frequency (right panel upper curves). Upper panels: short path; middle panels: long path; lower panels: short+long paths.

QPI and the phase modulation but only the QPI is robust and visible when spectrally averaged. Note finally that these simulations have been carried out with a low intensity dependence of $A_{q}^{(j)}$ (which is the case for plateau harmonics). A slight increase in the power dependence (harmonics getting closer to the cutoff) washes out the modulations induced by the temporal phase modulation: since the harmonic emission gets shorter, it is more confined close to the maximum of the laser envelope, i.e., between the two inflexion points, and there is not enough harmonic energy after the inflexion points to induce interferences.

In conclusion, the spectral filtering allows getting rid of temporal averaging, leading to a highly contrasted QPI. However, it may expose to spurious effects induced by phase modulation. To rule out the latter, it is necessary to check that the modulations survive to spectral integration (even though with a reduced contrast).

\section{B. Macroscopic response}

The spatial averaging effects in the macroscopic response pose an even bigger challenge than the temporal averaging. In order to simulate them, we have used a numerical model already described in [32]. Briefly, this two-dimensional code in cylindrical geometry solves the coupled propagation equations of the fundamental and harmonic fields in the paraxial 
and slowly varying envelope approximations. The source term in the propagation equation of the harmonic field, i.e., the induced nonlinear polarization, is calculated nonperturbatively within the SFA [12]. The optical field ionization of the gas is described by the Ammosov-Delone-Kraïnov tunneling model [33]. Both the depletion of the medium and the free-electron dispersion are included in the propagation equations, as well as the atomic dispersion and the absorption of the harmonic field. We consider a spatiotemporally Gaussian Ti:sapphire laser pulse, with a confocal parameter $b=1.2 \mathrm{~cm}$ and a $30 \mathrm{fs}$ FWHM pulse duration. The generating medium is modeled by a Lorentzian density profile of $L=1 \mathrm{~mm}$ FWHM truncated at $20 \%$ of the peak density. If not specified, the argon gas peak pressure is 10 Torr.

This loose focusing geometry $(b \gg L)$ is expected to limit spatial averaging in the longitudinal direction. However, transverse averaging is strong enough to smear out the QPI in the total harmonic signal, as shown by the dashed line in Fig. 3(b). To observe the QPI, we may use the spatial analog of the temporal-spectral filtering. The intensity dependence of the dipole phase leads to a curvature of the harmonic phase front (equivalent to a spatial chirp) $[15,26,28,34]: \phi_{q}^{(j)}(r, t) \approx-\alpha_{q}^{(j)} I(r, t)$. The phase front curvature of the long path contribution is larger than that of the short path contribution; this leads to fringes in the far-field distribution. Integrating the signal obviously smears out the QPI modulations, but a far-field spatial selection may prevent this transverse averaging. However, an additional difficulty arises here due to different phase matching of the two quantum paths [35]. We obviously need generation conditions where both paths give significant contributions to the macroscopic response. This implies focusing the laser after the generating medium. In this situation, the short path is phase matched on axis, and the long path is phase matched off axis. This results in a concentration of the short (long) path contribution at the center (outskirts) of the far-field profile. The relative contribution from the two trajectories thus varies radially. A far-field spatial filter selecting a single fringe and positioned off axis in order to balance the two contributions will thus maximize the QPI contrast.

To simulate this, we calculate the far-field harmonic field distribution and then we select the off-axis part, between 14 and $20 \mathrm{mrad}$ in the horizontal plane and between -0.5 and $+0.5 \mathrm{mrad}$ in the vertical one, in order to take into account the angular acceptance of the collecting mirror used in the experiment described in the next section. The filtered harmonic signal [solid line in Fig. 3(b)] shows clear oscillations due to QPI, although with a reduced contrast due to the remaining temporal averaging. We then calculated the spectrum of the spatially filtered harmonic emission. We show in Fig. 6 the evolution of the spectral shape of the 21st harmonic with intensity, calculated for different laser focus positions and filtering conditions. The spectral intensity is plotted on a logarithmic scale, with the color/gray scale being normalized to the maximum spectral intensity. The laser intensity corresponds to the peak intensity in the middle of the gas jet. This is the value calculated in vacuum, but the actual peak intensity may be somewhat lower close to the saturation intensity due to the onset of laser defocusing. Figure 6(a) corresponds to the case where the laser is focused $3 \mathrm{~mm}$ in
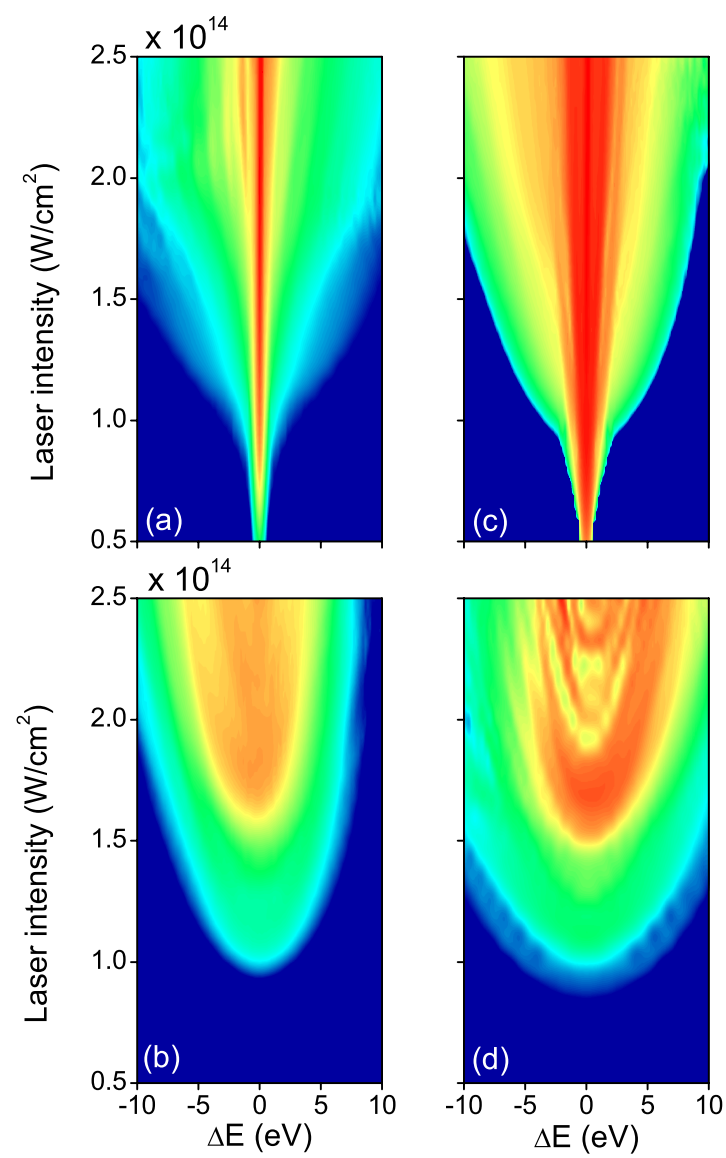

FIG. 6. (Color online) Calculated H21 spectrum as a function of the laser intensity for different focus and spatial filter positions. The spectral intensity is plotted on a logarithmic scale; the color/gray scale is normalized to the maximum spectral intensity. The laser intensity is given in the middle of the gas jet. (a) The laser is focused $3 \mathrm{~mm}$ in front of the gas jet and no spatial filtering is applied. (b) Same focus position as (a) with spatial selection in the far-field profile between 14 and $20 \mathrm{mrad}$ in the horizontal plane, and between -0.5 and $+0.5 \mathrm{mrad}$ in the vertical one. (c) Focus position: $3 \mathrm{~mm}$ behind the jet, without filtering. (d) Same focus position as (c); filtering is on.

front of the jet. No spatial filtering is applied. The spectrum is narrow $\left(\Delta \lambda / \lambda \approx 10^{-2}\right.$ at $\left.I=2 \times 10^{14} \mathrm{~W} / \mathrm{cm}^{2}\right)$ with a maximum on the spectral axis (i.e., detuning $\Delta E=0$ from the central harmonic frequency). This is a typical behavior of the short trajectory which contributes dominantly in this focusing condition and is spatially located on the laser axis. When an off-axis spatial selection is performed in the far field [Fig. 6(b)], the spectrum broadens, but the harmonic yield is not modulated. Figure 6(c) displays the case of focusing $3 \mathrm{~mm}$ behind the jet and without filtering. By comparison with Fig. $6(\mathrm{a})$, there is a clear increase in the off-spectral-axis strength, coming from the long trajectory, which contributes significantly to the emission in this focusing condition. Applying an off-axis spatial filtering in the far-field harmonic profile leads to a spectral broadening [18] and to a fringe pattern with a parabolic shape [Fig. 6(d)] and an on-axis periodicity of $0.3 \times 10^{14} \mathrm{~W} / \mathrm{cm}^{2}$. As described in the previous section, this spectral broadening is due to the dipole phase modula- 


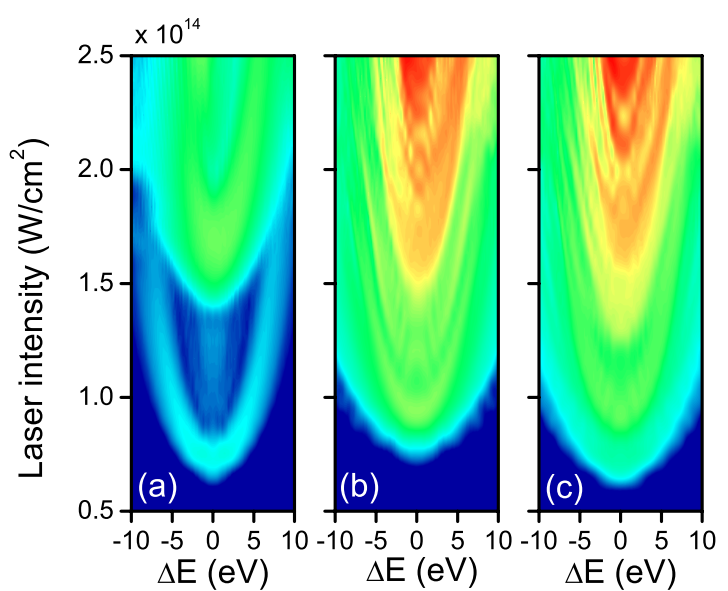

FIG. 7. (Color online) H21 spectrum as a function of laser intensity calculated using the saddle-point method and in the conditions of Fig. 6(d). (a) Short trajectory. (b) Long trajectory. (c) Short+long trajectories. The color scales are normalized to the maximum spectral intensity of case (c).

tion with laser intensity that induces a chirp on the harmonic emission. This effect is stronger on the long trajectory $(j=2)$ since $\alpha_{q}^{(2)}>\alpha_{q}^{(1)}$ (see Fig. 1). Note that the chirp depends on the lateral position in the beam through the spatial intensity profile. Thanks to the spatial filtering, we get very similar spectra as the single-atom spectra reported in Fig. 4(b), and in particular the parabolic shape of the interference.

To disentangle the respective contributions of the phase modulation and of the QPI, it is interesting to simulate the macroscopic spectra corresponding to individual trajectories. To do so, we used the single-path dipoles calculated within the saddle-point approximation. In Fig. 7, we report the evolution with intensity of the (filtered) spectra in the same conditions as in Fig. 6(d). The color scales are normalized to the maximum spectral intensity of Fig. 7(c). This explains why the signal corresponding to the short trajectory [Fig. 7(a)] is so weak. This low signal is due to the off-axis selection that cuts most of the short trajectory contribution that is concentrated on axis. In the spectra corresponding to the individual trajectories, we observe spectral broadening and structures due to phase modulation. For the short trajectory, the two maxima observed in Fig. 7(a) are spaced by 0.8 $\times 10^{14} \mathrm{~W} / \mathrm{cm}^{2}$ on the spectral axis, which is much larger than the QPI periodicity. Modulations which appear in Fig. 7(b) have no clear periodicity. A well-defined periodicity only appears when both trajectories contribute to the emission.

One crucial test to discriminate QPI from phase modulation is to spectrally integrate the signal in Fig. 7. In this case, we expect a disappearance of the fringes due to phase modulation since this corresponds to a frequency redistribution, whereas modulations due to QPI should survive the integration. The results of the integration are plotted in Fig. 8 as a function of the laser peak intensity. Modulations subsist only when the contributions of both trajectories are taken into account in the dipole (solid line). When only one of them is involved in the emission process, no modulation with clear periodicity survives frequency integration, as can be seen on

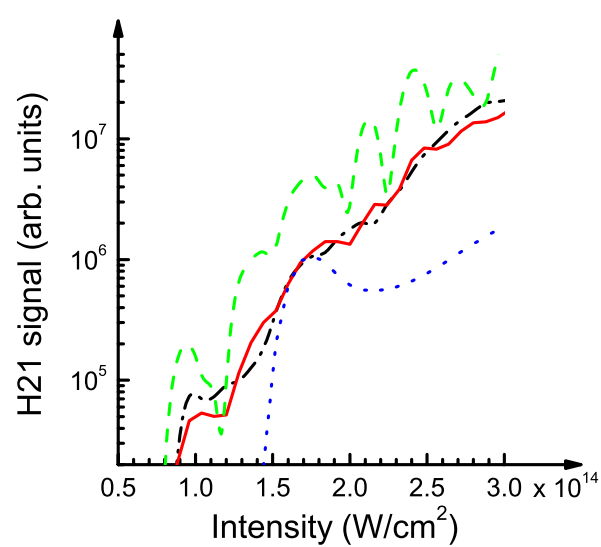

FIG. 8. (Color online) Spectrally integrated signal derived from numerical data given in Fig. 7: short trajectory (dotted line), long trajectory (dashed-dotted line), and short+long trajectories (solid line). For comparison, the dashed line gives a line cut at the central frequency of Fig. 7(c).

the dashed-dotted and the dotted lines. The dashed line depicts the evolution of the signal on the spectral axis $(\Delta E$ $=0$ ) when both short and long trajectories contribute to the harmonic emission. The comparison with the frequency integrated signal shows a much larger contrast due to the absence of temporal averaging and a small shift of the oscillations toward lower intensities, which is probably due to the fact that the frequency integration produces a mixing of different intensity contributions in the driving pulse temporal envelope, whereas the signal on the spectral axis coincides with the maximum of the temporal envelope. We thus conclude that even a small contribution of the short trajectory allows one to produce QPI.

Finally, it is interesting to compare the result obtained with the short+long trajectories (dashed line in Fig. 8) to that obtained when higher-order trajectories are taken into account (dashed line in Fig. 10). QPI involving only the two main trajectories lead to quasisinusoidal modulations whereas more complex structures (like double peaks) develop when using higher-order trajectories in the dipole calculation, especially for laser intensity larger than 1.9 $\times 10^{14} \mathrm{~W} / \mathrm{cm}^{2}$, as already mentioned. This comparison suggests that higher-order trajectories may also contribute to the modulation of the harmonic signal and thus would be observable in the macroscopic response. However, the main contribution below $1.9 \times 10^{14} \mathrm{~W} / \mathrm{cm}^{2}$ for the $21 \mathrm{st}$ harmonic, and on the whole intensity range for the 15 th, apparently stems from QPI between the two first trajectories.

The QPI behavior is very similar for the different harmonic orders, as shown in Fig. 9 giving a comparison of the 15 th and the 21 st harmonics. The fringe pattern is mainly shifted toward higher intensities with increasing order (corresponding to the shift in cutoff position), as can be seen in Fig. 10 giving a line cut at the central harmonic frequency $(\Delta E=0)$. This is consistent with QPI; indeed, the $\alpha$ values depend weakly on harmonic order in the plateau region and are only shifted in intensity. This can be easily shown by a Gabor-type analysis of the dipole (see Fig. 1) for different harmonic orders. We note that the spectra of both the 15 th 


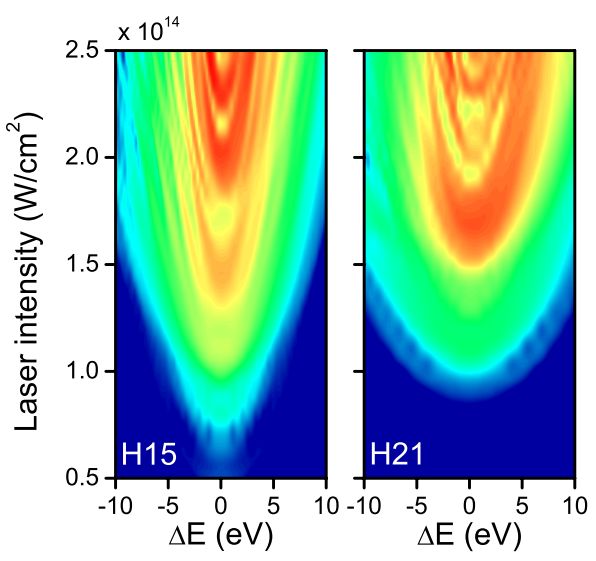

FIG. 9. (Color online) Calculated dependence of QPI on harmonic order. The simulations are performed with the full SFA dipole and in the conditions of Fig. 6(d).

and the 21st harmonic in Fig. 9 become asymmetric for intensities larger than $1.5 \times 10^{14} \mathrm{~W} / \mathrm{cm}^{2}$. When getting closer to the saturation intensity, depletion of the medium starts to play a role and mainly affects the harmonic emission on the trailing edge of the laser pulse. Due to the harmonic chirp, this corresponds to the red wing of the spectrum. Additional distorsions are also induced by the increasing electron dispersion: harmonic phase mismatch, laser self-phase modulation, and refraction.

In order to study the influence of the free-electron dispersion, we calculated the filtered harmonic spectra for different pressures of the generating medium. This is shown in Fig. 11 for pressures of 1, 5, and 10 Torr. The spectral shape as well as the periodicity is independent of the gas pressure. This is clearly evidenced in Fig. 12 showing the signal on the spectral axis derived from Fig. 11. Surprisingly, the fringe visibility increases with pressure. This is likely due to a change in phase matching with pressure that modifies the harmonic farfield distribution, especially for the long trajectory. Finally, we note that the weak influence of the medium pressure on the observed fringes rules out the ionization-induced selfphase modulation of the generating laser as a mechanism to produce these fringes.

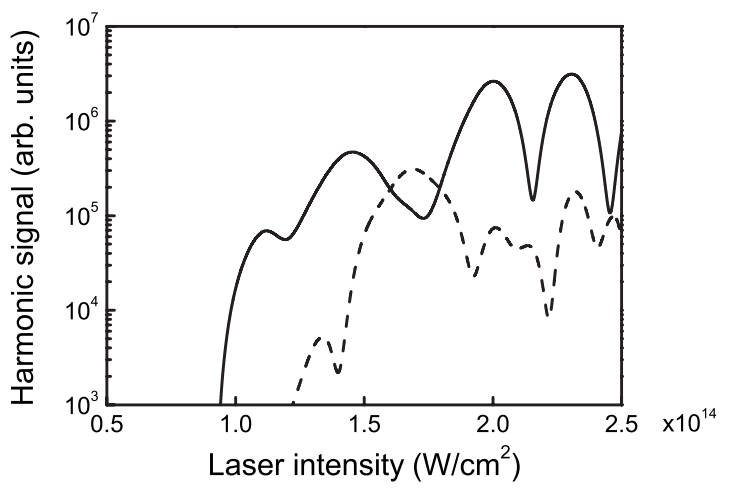

FIG. 10. Line cut at the central frequency $(\Delta E=0)$ from the fringe patterns given in Fig. 9: H15 (solid line), H21 (dashed line).

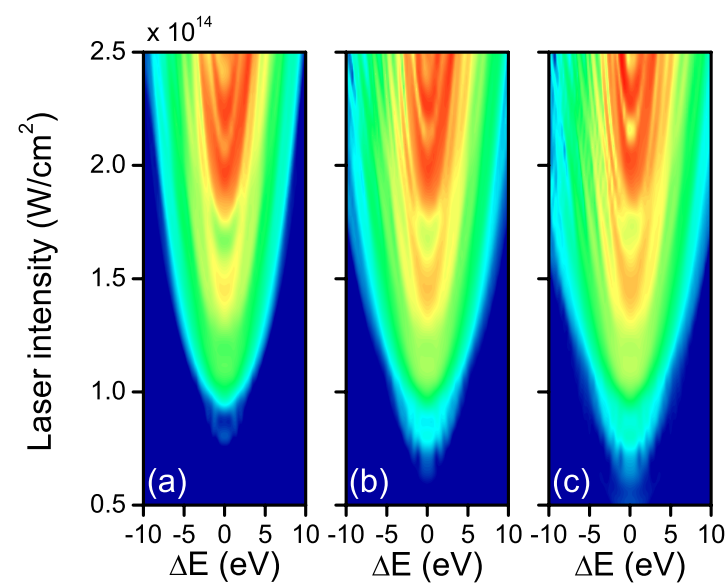

FIG. 11. (Color online) Dependence of QPI with pressure calculated for H15. (a) $P=1$ Torr, (b) $P=5$ Torr, and (c) $P$ $=10$ Torr.

\section{EXPERIMENT}

The experimental investigation is performed using a Ti:sapphire laser system delivering $30 \mathrm{fs} 800 \mathrm{~nm}$ pulses at a repetition rate of $1 \mathrm{kHz}$ with a maximum pulse energy of 1.5 $\mathrm{mJ}$. The setup used to observe the first-order QPI in argon is presented in Fig. 13.

The laser beam is focused by a $50 \mathrm{~cm}$ radius of curvature (ROC) spherical mirror into a pulsed argon jet exiting from a $300 \mu \mathrm{m}$ nozzle. The confocal parameter (defined as twice the Rayleigh range) is $1.2 \mathrm{~cm}$. The laser is focused at such a distance from the nozzle outlet that the medium length is 1 $\mathrm{mm}$. Unless specified otherwise, the backing pressure is 2.9 bar. A half-wave plate and a polarizer are placed in the infrared (ir) beam to precisely and continuously control the peak intensity in the jet from $0.5 \times 10^{14}$ to 4.5 $\times 10^{14} \mathrm{~W} / \mathrm{cm}^{2}$. A 150 -nm-thick aluminum filter is placed behind the jet to filter out the residual ir laser light. The high-harmonic emission is reflected by a gold spherical mirror $(\mathrm{ROC}=30 \mathrm{~m})$ under grazing incidence to the entrance slit of a spectrometer composed of a platinum grating and a backside-illuminated charge-coupled-device camera. The gold-coated mirror can be transversally moved from perfect on-axis alignment to an off-axis position, spatially selecting

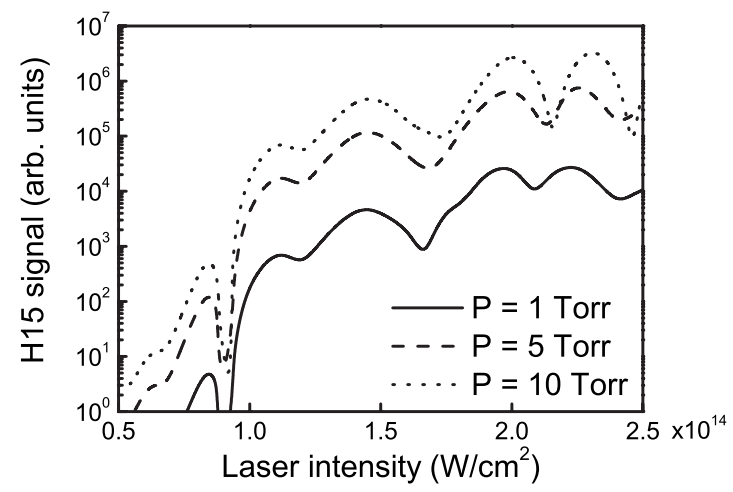

FIG. 12. Line cut at the central frequency $(\Delta E=0)$ from the fringe patterns given in Fig. 11. 


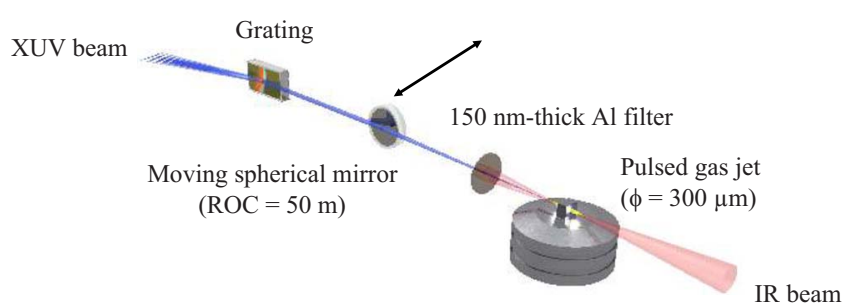

FIG. 13. (Color online) HHG experimental setup used for the observation and measurement of QPI.

part of the HHG beam, thus acting as a far-field spatial filter with an acceptance angle in the horizontal plane of 6 mrad. The slit of the spectrometer provides additional spatial filtering. We acquired each harmonic spectrum over 5000 laser shots. Note that our laser energy stability is high enough to ensure that QPI is not smeared out by intensity fluctuations. The peak laser intensity at the center of the jet was estimated from the intensity at focus measured by recording the focal spot size, the pulse duration measured by spectral phase interferometry for direct electric-field reconstruction, and the pulse energy. The experiment consists in recording harmonic spectra for different laser peak intensities with an on- or an off-axis spatial selection in the far-field $(6 \mathrm{mrad}$ spatial filtering). The peak laser intensity in the plots below is that reached in the middle of the jet.
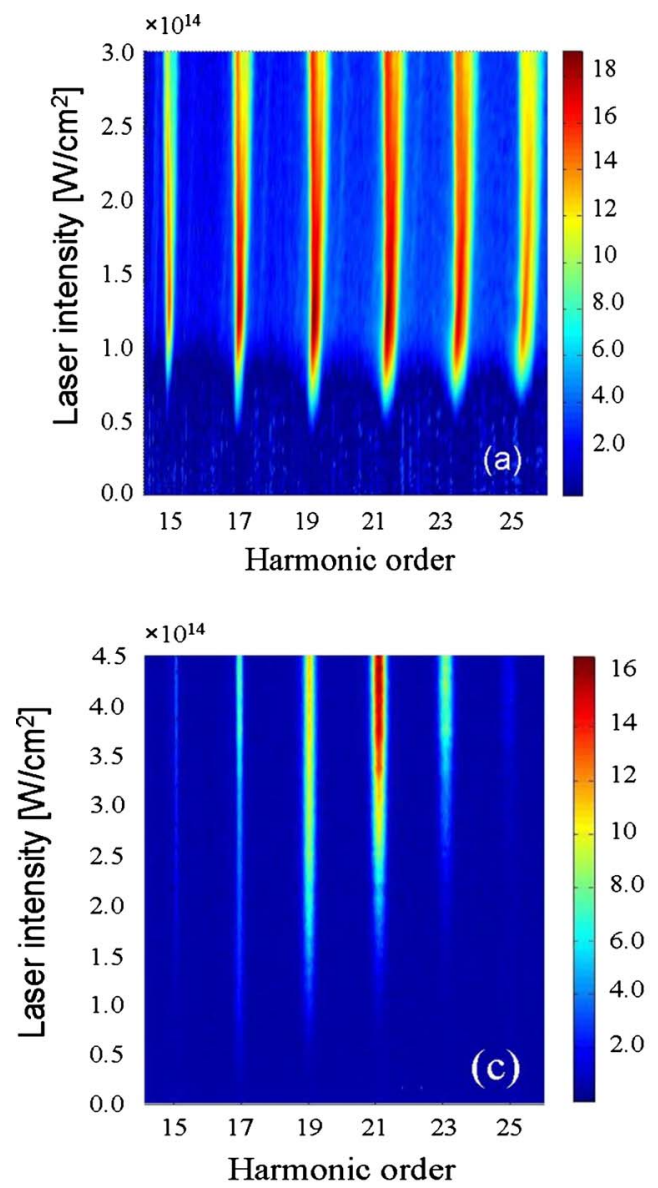

We first studied the influence of the laser focus position and of the spatial filtering. The objective was to check that there was no modulation of the harmonic amplitudes when selecting a single trajectory contribution, and that in the presence of many trajectories the spatial position and the width of the filter allowed one to resolve the QPI with a high enough contrast to evaluate the modulation periodicity and therefore the trajectories involved in the HHG process.

We first positioned the argon jet $3 \mathrm{~mm}$ behind the laser focus. In that case only the short trajectory is phase matched on axis. When we perform the on-axis selection [Fig. 14(a)] or the off-axis selection [Fig. 14(b)], the harmonics are spectrally narrow, although with broad but weak shoulders in the latter case. The harmonic amplitudes do not exhibit any modulations with laser peak intensity. This behavior is in good agreement with the fact that there is only one trajectory involved in the HHG process. Note that from the on- to the off-axis selection the plateau harmonic amplitudes decrease by one order of magnitude. In a second step, the jet is positioned $3 \mathrm{~mm}$ in front of the laser focus where the two first quantum paths are phase matched. When performing the onaxis selection, the short trajectory dominates due to its smaller divergence. As expected, the harmonics are spectrally narrow and without modulations [Fig. 14(c)]. When an off-axis selection is performed [Fig. 14(d)], a clear broadening of the plateau harmonics (from order 13 to 25) is ob-
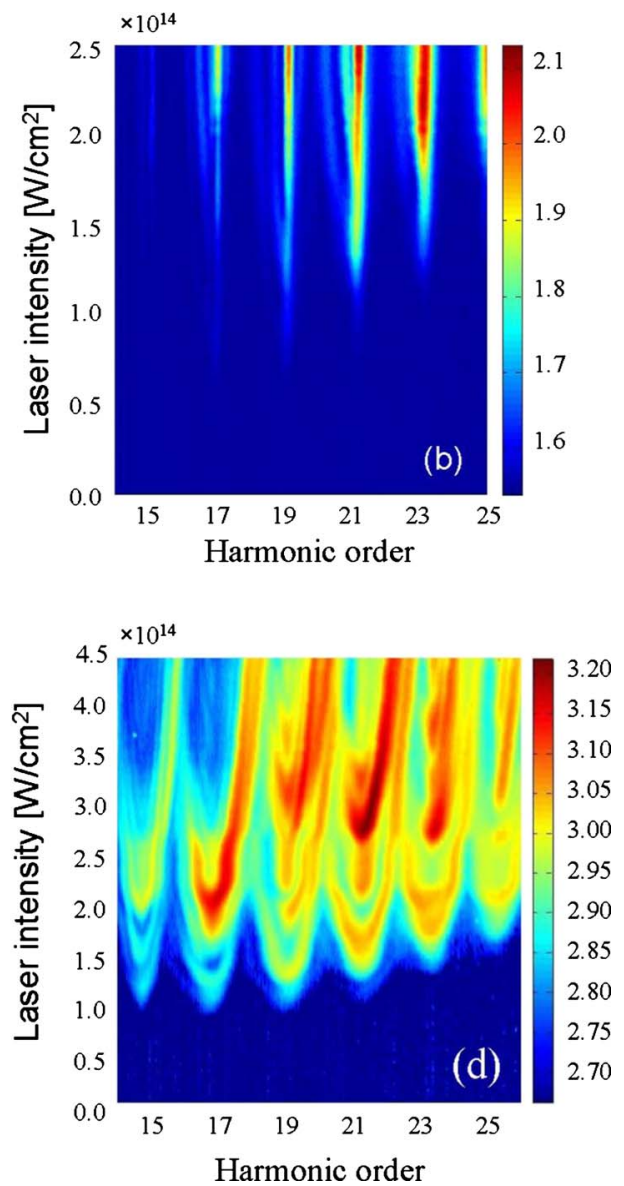

FIG. 14. (Color online) Measured harmonic spectrum as a function of laser peak intensity for 6 mrad far-field spatial filtering: (a) and (b) jet positioned $3 \mathrm{~mm}$ behind the laser focus and (a) on-axis filtering or (b) off-axis filtering. (c) and (d) jet positioned $3 \mathrm{~mm}$ in front of the laser focus and (c) on-axis filtering or (d) off-axis filtering. 
served due to the phase modulation of the long trajectory. Moreover, spectral modulations with a parabolic spectral shape clearly appear. This is in good agreement with the numerical results presented in Fig. 6(d). At high intensity, neighboring harmonics start to overlap in such a way that the high frequency part (blue component of the long path contribution) of a harmonic $q$ may interfere with the low frequency part (red component of the long path contribution) of a harmonic $q+2$. The red component is weaker than the blue due to the depletion of the medium, as observed in Fig. 9. In our experiment, the laser CEP was not stabilized [36-38]. This is the reason why we do not observe interferences between long path contributions from neighboring harmonic orders when they overlap at even harmonic frequencies [17]. Indeed, since the CEP was random, these interferences are smoothed out in the averaging over 5000 shots and do not disturb the observation of the QPI. QPI observation does not require the CEP stabilization since it occurs on the subcycle attosecond time scale. At the central harmonic frequency we observe plateau-harmonic yield modulations as a function of peak laser intensity. The modulation periodicity $(\sim 0.3$ $\times 10^{14} \mathrm{~W} / \mathrm{cm}^{2}$ at the harmonic central frequency) coincides with the calculated value for the first-order QPI.

Finally, in order to show that the QPIs observed are not pressure dependent, we have measured the high-order harmonic spectra for high (2.9 bar) and low (1.45 bar) backing pressures. As in Fig. 14(d), the jet is positioned in front of the focus and the selection is performed off axis. The results are shown in Fig. 15 for H21. From high to low pressure, the harmonic amplitude decreases roughly by a factor of 2 , so the harmonic starts to be detected at higher intensity than in the high-pressure experiment. However, the shape of the harmonic and the QPI, in particular the periodicity at the central frequency, do not depend on pressure, in good agreement with the numerical results in Fig. 12.

\section{CONCLUSION}

In summary, we have found experimental conditions where the QPI are observable in the macroscopic response: this is obtained through optimized phase matching, spatial, and spectral filtering. We reported thorough calculations that identify unambiguously the origin of the intensity modulations observed in the experimental high-harmonic spectra: they are produced by the interference of the contributions of the two shortest electron quantum paths. Our simulations also indicate that higher-order quantum paths may contribute to the fringe pattern close to the saturation intensity, by
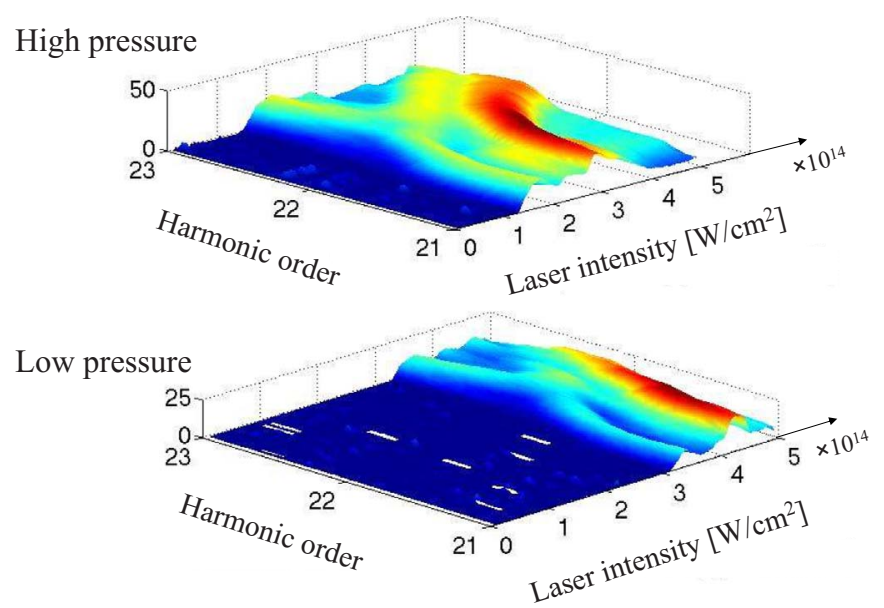

FIG. 15. (Color online) Measured H21 spectrum as a function of laser peak intensity with $6 \mathrm{mrad}$ off-axis spatial filtering in the far field. The jet is positioned $3 \mathrm{~mm}$ in front of the laser focus. (Upper) High pressure (2.9 bar backing pressure); (lower) low pressure (1.45 bar backing pressure).

modifying the fringe periodicity. Our experiments are thus a step toward the direct measurement of the full single-atom dipole. Moreover, the laser intensity dependence of the relative phase of the quantum path contributions enables one to control their interference pattern. This "atomic interferometer" allows controlling the duration of each trajectory on an attosecond time scale. Indeed, at $1.5 \times 10^{14} \mathrm{~W} / \mathrm{cm}^{2}$, a $10 \%$ variation in the intensity shifts the QPI from constructive to destructive interference for the 15 th harmonic. Saddle-point calculations show that the corresponding variations in excursion times are extremely small for both quantum paths: $\Delta \tau_{15}^{(1)}=-22$ as and $\Delta \tau_{15}^{(2)}=+13$ as (see Fig. 2).

\section{ACKNOWLEDGMENTS}

This research was supported by the NCCR Quantum Photonics (NCCR QP), research instrument of the Swiss National Science Foundation (SNSF), the Research Councils UK through the UK Attoscience Consortium (Grant No. EPSRC GR/S24015/01), the French Agence Nationale de la Recherche (Grant No. ANR-05-BLAN-0295-01, ATTOSCIENCE), Grant No. COST-STSM-P14-01910, the European Commission through the RTN XTRA (Grant No. MRTN-CT-2003-505138), and the M3PEC-UBx1 computer center funded by the Conseil Régional d'Aquitaine. The authors would like to thank A. L'Huillier, G. Sansone, and M. B. Gaarde for stimulating discussions.
[1] P. Agostini and L. F. DiMauro, Rep. Prog. Phys. 67, 813 (2004).

[2] P. M. Paul et al., Science 292, 1689 (2001).

[3] Y. Mairesse et al., Science 302, 1540 (2003).

[4] Y. Mairesse et al., Phys. Rev. Lett. 93, 163901 (2004).

[5] M. Hentschel et al., Nature (London) 414, 509 (2001).
[6] G. Sansone et al., Science 314, 443 (2006).

[7] P. Johnsson et al., Phys. Rev. Lett. 95, 013001 (2005).

[8] M. Uiberacker et al., Nature (London) 446, 627 (2007).

[9] W. Boutu et al., Nat. Phys. 4, 545 (2008).

[10] P. B. Corkum, Phys. Rev. Lett. 71, 1994 (1993).

[11] K. J. Schafer, B. Yang, L. F. DiMauro, and K. C. Kulander, 
Phys. Rev. Lett. 70, 1599 (1993).

[12] M. Lewenstein, P. Salières, and A. L'Huillier, Phys. Rev. A 52, 4747 (1995).

[13] P. Salières et al., Science 292, 902 (2001).

[14] Ph. Balcou, A. S. Dederichs, M. B. Gaarde, and A. L'Huillier, J. Phys. B 32, 2973 (1999).

[15] M. B. Gaarde, F. Salin, E. Constant, Ph. Balcou, K. J. Schafer, K. C. Kulander, and A. LHuillier, Phys. Rev. A 59, 1367 (1999).

[16] J. Mauritsson, P. Johnsson, R. Lopez-Martens, K. Varju, W. Kornelis, J. Biegert, U. Keller, M. B. Gaarde, K. J. Schafer, and A. LHuillier, Phys. Rev. A 70, 021801(R) (2004).

[17] G. Sansone, E. Benedetti, J. P. Caumes, S. Stagira, C. Vozzi, S. De Silvestri, and M. Nisoli, Phys. Rev. A 73, 053408 (2006).

[18] E. Benedetti et al., Opt. Express 14, 2242 (2006).

[19] C. Corsi, A. Pirri, E. Sali, A. Tortora, and M. Bellini, Phys. Rev. Lett. 97, 023901 (2006).

[20] H. Merdji, M. Kovacev, W. Boutu, P. Salieres, F. Vernay, and B. Carre, Phys. Rev. A 74, 043804 (2006).

[21] J. J. Macklin, J. D. Kmetec, and C. L. Gordon, Phys. Rev. Lett. 70, 766 (1993).

[22] C.-G. Wahlström, J. Larsson, A. Persson, T. Starczewski, S. Svanberg, P. Salieres, Ph. Balcou, and A. L'Huillier, Phys. Rev. A 48, 4709 (1993).

[23] A. Zair et al., Phys. Rev. Lett. 100, 143902 (2008).

[24] G. Sansone, C. Vozzi, S. Stagira, and M. Nisoli, Phys. Rev. A
70, 013411 (2004).

[25] M. Lewenstein, Ph. Balcou, M. Yu. Ivanov, A. L'Huillier, and P. B. Corkum, Phys. Rev. A 49, 2117 (1994).

[26] P. Salières, A. L'Huillier, and M. Lewenstein, Phys. Rev. Lett. 74, 3776 (1995).

[27] Z. Chang, A. Rundquist, H. Wang, I. Christov, H. C. Kapteyn, and M. M. Murnane, Phys. Rev. A 58, R30 (1998).

[28] P. Salières et al., Adv. At., Mol., Opt. Phys. 41, 83 (1999).

[29] K. Varju et al., J. Mod. Opt. 52, 379 (2005).

[30] K. Varju et al., Phys. Rev. Lett. 95, 243901 (2005).

[31] P. Salières, Ph. Antoine, A. de Bohan, and M. Lewenstein, Phys. Rev. Lett. 81, 5544 (1998).

[32] A. L'Huillier, Ph. Balcou, S. Candel, K. J. Schafer, and K. C. Kulander, Phys. Rev. A 46, 2778 (1992).

[33] M. V. Ammosov, N. B. Delone, and V. P. Kraïnov, Sov. Phys. JETP 64, 1191 (1986).

[34] C. Lyngå, M. B. Gaarde, C. Delfin, M. Bellini, T. W. Hänsch, A. L'Huillier, and C. G. Wahlström, Phys. Rev. A 60, 4823 (1999).

[35] Ph. Balcou, P. Salieres, A. LHuillier, and M. Lewenstein, Phys. Rev. A 55, 3204 (1997).

[36] H. R. Telle et al., Appl. Phys. B: Lasers Opt. 69, 327 (1999).

[37] D. J. Jones et al., Science 288, 635 (2000).

[38] A. Apolonski, A. Poppe, G. Tempea, C. Spielmann, T. Udem, R. Holzwarth, T. W. Hansch, and F. Krausz, Phys. Rev. Lett. 85, 740 (2000). 